\title{
WORKPLACE VIOLENCE: A QUALITATIVE STUDY DRAWING ON THE PERSPECTIVES OF UK NURSING STUDENTS
}

\begin{abstract}
Background: Workplace violence (WV) remains problematic and highly prevalent in healthcare with student nurses being especially vulnerable.
\end{abstract}

Aim: To investigate the impact of WV from the perspectives of nursing students

Methods: A qualitative design was employed. 444 nursing students in the UK participated in the study.

Findings: The qualitative data was analysed, coded and three main themes have emerged; (1) violence culture in nursing, (2) tolerating violence and (3) the impact of the violence.

Discussion: The undesirable truth is each year nearly more than half of the students face WV in environments where such behaviour becomes culturally embedded and passed between generations of nurses. This process of normalization requires intervention that will break this cycle to enable more appropriate workplace behaviours to flourish.

Conclusion: The impact of WV can be extremely destructive for the individual, the profession and ultimately patient care with large numbers choosing to leave at a time when there is a national shortage of qualified staff.

Key Words: Nursing, nursing students, violence, workplace, workplace violence, 
Summary of relevance

Problem

WV undermine safe work environments and potentially lead to physical-psychological problems amongst targets of workplace violence

\section{What is already known}

Internationally, student nurses are at risk of being targeted and bullied during clinical practice.

\section{What this paper adds}

The findings provide a deeper understanding of the WV on nursing students, which can help to identify effective solutions that actively address the issues. 


\section{INTRODUCTION}

Workplace violence (WV), is often described as 'violence directed toward workers that can include physical, sexual, psychological or verbal acts' (Gacki-Smith, Juarez, Boyett, Homeyer, Robinson, \& MacLean, 2009). It has been characterized as an insidious, personalized and harmful experience and is receiving growing attention in the literature (Tee, Üzar-Özçetin, \& Russell-Westhead, 2016; Birks, Cant, Budden, Russell-Westhead, Üzar Özçetin, \& Tee, 2017).

WV remains problematic and highly prevalent in healthcare (Berry, Gillespie, Gates, \& Schafer, 2012; Hartin, Birks, \& Lindsay, 2018; Tee et al., 2016) with student nurses being especially vulnerable. Studies suggest from a perpetrator perspective the causes can be multiple, but typically include impaired personal relationships, lack of experience, powerlessness, lowered self-esteem and understaffing causing high workload (Islam, Ahmed, \& Ali, 2019; Mammen, Hills, \& Lam, 2018). Similar studies show that student nurses may frequently be the targets due to their younger age, less job experience and lower status in the hierarchy (Mammen et al., 2018; Sun, Ge, Ke, Li, Jin, \& Lu, 2018; Tee et al., 2016). Those targeted can experience emotional reactions such as aggression, anxiety, irritability, apathy, fatigue, lowered morale, and hopelessness (Avander et al., 2016; Hills, Lam, \& Hills, 2018; Birks et al., 2017). WV also has negative impacts on professional skills and patient care. It affects negatively nurses' ability to handle or manage their workload, to communicate or to cope effectively and raises their stress levels (Berry et al., 2012), which can lead to low ratings of patient care quality. Perhaps most significantly, WV has a direct result on staff retention with those targeted as expressing the intent to leave the profession (Fahie, \& McGillicuddy, 2018; Wressell, Rasmussen, \& Driscoll; 2018). Studies (Birks et al., 2017; Mammen et al., 2018; Tee et al., 2016; Üzar-Özçetin, Tee, \& Erkan, 2020) reports suggest a third of new nurses, who experience workplace bullying, intend to leave their current position. 
Such behaviours undermine safe work environments and potentially lead to physicalpsychological problems amongst student nurses (Ahn \& Choi, 2019; Minton, Birks, Cant, \& Budden, 2018; Tee, Üzar Özçetin, \& Russell-Westhead, 2016; Üzar-Özçetin et al., 2020) as well as impacting negatively on the quality of patient care (Avander, Heikki, Bjerså, \& Engström, 2016). It is recognized that most qualified nurses and nurse educators invest considerable time and effort in student learning to provide a positive experience for students to learn and achieve the necessary competence (Emanuel \& Pryce-Miller, 2013). However, student nurses too often express dissatisfaction in placement, citing bullying and harassment as one of the causes. Nursing students are valuable to the profession and more needs to be done to empower them whilst on placement (Glomsås, Tranum, \& Johannessen, 2019; Jenkins, \& Germaine, 2018; Manankil-Rankin, Lunyk Child, Chen, Martin, \& Bentley Poole, 2016; Tee et al., 2016; Tee, Üzar-Özçetin, Russell-Westhead, 2017).

Given the importance of this issue for the reputation of the profession and the nursing workforce in the UK. Prevention of WV is essential and this study sought to understand the student nurses' perspective that might help to ameliorate the problem. This study formed one

phase of a larger study by Tee, S., Üzar Özçetin, Y. S., \& Russell-Westhead, M. (2016), which reported on prevalence. This phase reports on the qualitative data which examined the effects of WV from the perspectives of nursing students perceived to be at risk of WV.

\subsection{Theoretical Framework}

In our approach to this study we were interested in understanding why certain workplace behaviours appear to be accepted and tolerated, despite the negative impact and consequences of such behaviours. To help frame the narrative we employed Normalization Process Theory (NPT) which develops understanding of the social processes that occur to allow new or modified practices of thinking and organizing workplace in healthcare settings (May \& Finch, 2009). NPT helps to explain how work-related behaviours become embedded and accepted 
into the day-to-day work (Gallacher, May, Montori, \& Mair, 2011, May \& Finch, 2009; Tee et al., 2016). May \& Finch (2009) emphasised that NPT provides an analytic framework for understanding structures and embedded practices and also provides tools for helping enact a new set of practices. This theory helped to structure and explain the findings.

\section{METHODS}

\subsection{Design}

A qualitative study design was used for this phase of the study, by adopting a phenomenological approach which is a philosophy and method for gaining an in-depth understanding of lived experience (Lopez \& Willis, 2004). This allows the researcher to become more aware of the interpretation of the encountered phenomena, in this case workplace violence (Mackey, 2004). Thus, Heidegger's phenomenological approach was used to guide this study. According to Heidegger, the views and lived experiences shape the meaning and help capture the meaning embedded in that experience (Heidegger, 2010). Heidegger' phenomenology requires that researchers identify participants' perceptions on a subject and apply their own interpretation of these perceptions. In effect, it allows researchers to interpret and analyze meanings and suppositions which participants find difficult to express (Laverty, 2003). In accordance with the Heidegger' phenomenological approach, with an extra question rather than semi-structured open ended questions, the students were asked whether they wanted to add anything about their experiences after they had completed the qualitative phase. The study also followed the COREQ Checklist (COREQ, 2019).

\subsection{Setting}

The study was conducted using the format of a commercial Internet survey provider (SurveyMonkey.com) to be able to access students across the UK. A purposive sample of UK Higher Education Institutions with nursing schools/faculties was identified and invitations to participate were sent to the Heads of Schools asking for their participation. Once agreed, an 
email was sent containing a brief explanation of the research and a link to the survey. The survey included both quantitative and qualitative phases and students were free to participate in both or in only one phase of the study. The first phase was quantitative, exploring prevalence, which comprised five sections with 66 individual items based around workplace violence including intimidation, bullying or verbal abuse, non-physical violence, and reporting and management of workplace violence. The number of respondents was 657 . This first phase of the study has been published (Tee et al. 2016). The second phase was qualitative and based on open-ended questions to explore impact, meaning and possible interventions. The number of respondents was 444. The phase involved collecting student's written responses to open-ended questions (see Fig 1).

\subsection{Sample}

A purposive sampling of nursing schools, in accordance with the study's aim, was used to recruit student nurses who had experienced clinical placements. The inclusion criteria were being of 18 years of age or older, having had experience of clinical placement and having the ability to provide informed consent. The total student population enrolled in a nursing degree in the UK across 72 schools of nursing is approximately 60,000 (Tee et al., 2016). Each school was labeled with a number and random numbers list was used to select the schools to be contacted. One-fifth $(n=15)$ of the total schools were randomly contacted. This translated into approximately 6000 potential respondents as the size of student population in each school was in some cases quite small. There 657 actual responses to the questionnaire of which 444 responded to the qualitative part.

\subsection{Data Collection}

On accessing the survey, the student participants were presented with a Participant Information Sheet (PIS). The PIS facilitated a process of informed consent by asking participants to confirm that they had read the statement. Consent was therefore assumed by 
their submission of the survey. 444 students agreed to participate and completed the openended questions whilst two hundred and thirteen students declined to participate in the qualitative phase of the study. There were no prior relationships between the participants and the researchers. A pilot test was employed with a small sample of students unrelated to the study to test the questions and to understand how long it would take to complete the process. The open-ended questionnaire took 30 minutes on average to complete. All answers were written in English. The information regarding the participants' demographics was obtained before commencing the semi-structured, pilot-tested, interview guide (Fig. 1) which was based on previous literature (Avander et al., 2016; Birks et al., 2017; Tee et al., 2016;).

\subsection{Data Analysis}

The data were downloaded from the SurveyMonkey system and exported to The Microsoft Word Office program. The researchers both of whom (YSÜÖ, MRW) are female and nurse educators with a Ph.D. followed a systematic analytical process (Neergaard, Olesen, Andersen, \& Sondergaard, 2009). First, the researchers individually examined each exported document by identifying the sentences reflecting the main idea of each diary. Then, researchers highlighted the sentences pertaining to the phenomenon under question. Next, the documents were initially read through to interpret what the phenomenon revealed and reviewed repeatedly to gain familiarity. Subsequently, each document was analyzed independently to ensure credibility and trustworthiness. Topics were determined to cluster similar ideas and codes were generated. Codes were put together and thematic coding was carried out to determine the themes. To ensure rigor, a third researcher (ST) looked over the emerged themes and where necessary referred back to the data to make a final judgment. The researcher (ST) is a male nurse educator with a Ph.D. degree, also a professor at a University. All researchers had experience in conducting qualitative research. Researchers reread and organized the data obtained through this system according to the codes and themes. The 
researchers were aware of possible unequal power dynamics between the participants and the researchers (Malterud, Siersma, Guassora, 2015), thus using the SurveyMonkey system help the researcher to prevent the possible anxiety of students while expressing their experiences as a result of unequal power dynamics.

\subsection{Ethical Considerations}

Ethical approval was obtained from the Research Ethics Committees (CREC) of Kings College London (PNM/14/15-28). The participants received an information sheet detailing the scope of the study and a link to the SurveyMonkey. Participation was entirely voluntary and students could choose not to participate or withdraw without any impact on them or their studies. No individually identifiable data was requested in the survey and where participants included any such data, such as names of organizations or individuals, all data was anonymised. All the written material and web-page copies generated during the study were stored in a locked safe.

\section{RESULTS}

\subsection{Sample}

There were approximately 60,000 students in the UK. This translated into approximately 6000 potential respondents, with 657 actual responses. Of the 657 respondents in the first phase of the research (Tee et al., 2016), $444(67.58 \%)$ agreed to participate in the qualitative phase. The present study existed in collecting student's written quotes based on open-ended questions (see Fig 1).

The participants' mean age was $26.34+/-7.88$ and the majority were female (88.3\%). Most were attending university in the southeast of the UK (45.7\%), $84.8 \%$ were UK-born, $75.6 \%$ of the students' ethnic group was white, and $84.6 \%$ of the students' first language was English (see Table).

\subsection{Themes}


The qualitative data was analysed, coded and grouped under three main themes and ten subthemes. These themes are (1) violence culture in nursing, (2) tolerating violence

(3) the impact of the violence (See Fig 2).

\subsubsection{Violence culture in nursing}

Student nurses are particularly vulnerable and can be strongly influenced by culture. Having less experience and typically being younger lowers students' status in the team and makes them potential targets for WV. They may even be targeted by more senior staff who make unreasonable demands, setting excessive expectations and not providing support or mentoring.

\subsubsection{Acceptance of violence}

Whilst recognizing there will be a teaching role that includes feedback on performance, it is the disrespectful manner which it is given that is at issue. It must be remembered that harassment and bullying policies suggest it is how the comment, behaviour is received not what was intended by the initiator. Many students felt vulnerable because of being 'student' and having no-one to defend them.

"It is especially younger students. Some people see them as easy prey."

"Sometimes we just get seen as "the student" and what we say doesn't always get taken seriously."

"I was disrespected on the basis that I am 'below' qualified staff and because I am a student." It seems that culturally there is an acceptance in the profession that students should follow a well-trodden path and expect to experience WV from their colleagues. One indicated:

"You're almost always certainly going to be harassed in some way, particularly with comments like 'the student can do it'",

"I've been repeatedly shouted at, criticized, belittled \& called names."

Individual characteristics may be targeted: 
"Passing comments over my age have been made like 'are you old enough to be training?" Or abilities: "A registered nurse mocked me for how long it took me to carry out the morning drugs round and just referred to me as 'the student'."

\subsubsection{Sitting on the side-lines}

Full emersion in clinical experience is vital for students to gain the necessary competence and confidence. Feeling part of the profession is essential for development. Nevertheless, many did not feel part of a team because of exclusionary behaviours. Not being given due consideration and not being listened to by nurses and mentors appears to make students feel angry and isolated: "Nurses and members of staff ignore me and do not involve me in conversations. "

"I have been left out of staff meetings and left to 'man' the ward."

The negative nature of such behaviours may make individuals avoid engagement and begin to prefer being separated from the team.

These and similar responses gave insight into the prevailing cultures:

"Bullied for challenging practice and making suggestions and put down for trying to be an active team member and caregiver and made fun of for this."

\subsubsection{Learning by humiliation}

Those responsible for clinical teaching have also used humiliating tactics in the process of learning. Student nurses described the impact of WV were typically negative and expressed feelings of frustration and powerlessness.

"I was verbally abused for being stupid and was humiliated during handover for my mistake. However, I had no bad intent and it was an honest mistake."

Students felt there was a lack of empathy amongst mentors. One student with a disclosed learning disability added; 
"My mentor has made fun of my learning differences. She also implied that my parents must be disappointed in me."

Some said it was their physical appearance that was criticized:

"My mentor told me I need to lose weight (I'm a size 10) when I couldn't get past clutter... at the side of a bedside when we were doing meds."

"I have had mentors make fun of my physical appearance with one even stating I looked like one of the bimbos her son brings home'."

Sadly, many nursing students had experienced humiliation across settings and they were understandably uncomfortable with gossip and hurtful comments toward them or their colleagues.

"There were Registered Nurses gossiping and making fun of another student at the nurses' station in front of staff and patients."

These were not isolated incidents:

"I have noticed on placements of staff generally talking down to students or making faces behind students back when they think no one can see." and suggested that professional boundaries were not always observed:

"I have been spoken about behind my back to other staff and staff would speak to each other about other students/staff/patients in front of me."

\subsubsection{Tolerating violence}

Workplace culture is directly influenced by the behaviours of the team and impacts on members' working relationships The culture thus becomes normalized and shared and when WV goes unchecked and is tolerated it becomes accepted as part of the culture.

\subsubsection{Normalizing Poor Behaviour}

Nurses who work in areas where WV is normalized may not be aware of their own tendency to display disrespectful behaviours. Thus, there is a risk that bullying, when characteristic of 
an 'oppressed group behaviour', perpetuates the notion that it is 'routine' and a 'normal' part of the experience of nursing.

There were many examples where students suggested WV was normal and to be expected;

"I think sometimes students don't complain as it just seems to be an accepted part of being a student and the way people are within the healthcare environment." and the prevailing culture can prevent investigation: "I felt as though the comment was not significant enough to be reported and if I had reported it, there would be hostility between me and the said staff member, which would make for an uncomfortable placement."

\subsubsection{Negative Role Models}

The negative role modeling exhibited by perpetrators was a further aspect of the culture being accepted as the norm. This negative modeling damages the development of students' competence.

"I have been ignored \& neglected by mentors."

Students observe their mentors, accept them as role models and learn through them. This role modeling may cause them to internalize the professional values negatively. Preparing the future generation of nurses in a professional working environment is essential, and those attitudes described above threaten student progression and retention.

"Mentors can be inappropriate at times expecting far too much and never praise when you do something right but are more than happy to point it out when something's wrong!"

\subsubsection{Losing Hope}

The reporting system is crucial for the immediate implementation of the necessary corrective actions. Students who were a target for WV, often believe responding is futile, even though they knew about the policies on bullying/harassment:

"Once you report a nurse as a bully the whole team of nurses in the ward will go against you, so sometimes it is best to keep your mouth shut." 
"My mentor later insisted that the incident had never occurred."

"Although though several members of staff complained they too had been bullied and witnessed the treatment I received, they were too afraid to speak out."

Even when incidents were reported participants did not have confidence in the process:

"I told my tutor however I wasn't sure if any action was taken because when I went back on to that placement in my second year I was still experiencing it."

\subsubsection{The impact of the violence}

The students reported that WV had a negative impact on their learning, their perceptions of the profession, their self-esteem and their ambition for the future.

\subsubsection{Diminished learning opportunities}

Whilst engagement and participation in the clinical workplace is extremely valuable to learn about real-world of nursing, students reported feeling punished and threatened with consequences for their learning:

"I was told that if I am told to jump, I should ask how high or I will get to my final placement and be failed. The trained nurses are friends and they talk to each other."

Students too often cite bullying and harassment as one of the causes of dissatisfaction with their training. "Threatened with placement fail grade for requesting a Saturday off after working 4 weekends. " Such unfriendly environments cause dissatisfaction:

“Reduces learning opportunities and makes you feel like you shouldn't be there." Also, it can make students mistrustful of the profession and colleagues.

\subsubsection{Effect on self-esteem and self-concept}

One of the most revealing aspects of this study is the impact of WV on individual well-being: Students nurses reported WV as destablising and upsetting and that it lowered self-esteem and their self-belief. 
"I feel like a student nurse it happens all the time and sometimes it makes you feel so worthless and has a massive impact on my self-confidence."

Students reported their feelings and mood were affected negatively due to the uncivil behaviours. One reported: "It made me feel uncomfortable and defensive."

Another stated: "Question my abilities and caused me great anxiety."

Students do not have a well-defined role and this makes them vulnerable. Thus, they can easily undermine violence to their self-esteem and morale. One student expresses their feelings: "So frustrated at how childish some staff was and just how unnecessarily horrible staff can be, I just don't understand it?"

The relentless and deliberate nature of WV has been known to cause not only psychological harm, but also physical illness, financial loss and, ultimately, an inability to work.

"Useless like nothing you do is correct when you're trying your best!"

\subsubsection{Giving $U p$}

The impact of WV was at times, so significant that some considered leaving the program. Intention to leave the profession is a consequence of feeling powerless and the belief that nothing with changes.

"I have sometimes been put in situations in which I feel are not suitable for a student. I have considered leaving many times, and my attendance has suffered."

Bullying behaviours also exist because of a white wall of silence that often protects the bully. The targets of WV can be labeled "troublemakers" and fear loss of career advancement. Such silence unfortunately often allows bullying to continue in the workplace.

"Has often made me feel inadequate and considered leaving the nursing profession and that it is not worthwhile."

\section{DISCUSSION}


The aim of this phase of a larger study into workplace violence (WV) was to determine the impact of WV from the perspectives of nursing students in a UK sample. We believe that the insights derived from this study will have wider relevance to countries facing similar challenges in meeting the growing problem with regards to WV toward nursing students.

Nursing is one of the oldest health care professions in the world and has the positive potential for developing a strong professional individual and team identity reflecting expectations of good standards of care, professionalism and high patient satisfaction. However, it can also have a dark, destructive side manifested through WV, which if unchecked can damage the reputation of the profession as well as causing individual harm (Pryde, 2014). It is important to acknowledge the considerable investment of time and effort mentors and teachers contribute to learning to ensure students achieve the necessary skills and confidence to practice once qualified (Becher \& Visovsky, 2012). Nursing rightly has a proud tradition of selfless commitment to people when they are at their most vulnerable, which includes students learning, and for providing high-quality patient care (Üzar-Özçetin et al., 2020; Tee et al., 2016). Sadly, there seem to be clinical learning environments that are letting the profession down. This study was initiated following student feedback indicating that people were leaving nursing courses because of poor experiences and lack of support in practice. They specifically cited bullying and harassment from qualified staff, including mentors, which led to this survey. It is somewhat alarming that the findings mirror studies undertaken over a decade ago and that employers and the profession do not seem to have fully addressed the problem.

WV causes anger, frustration, feelings of powerlessness, decreased morale and productivity and perhaps more worryingly for patient care, an increase in errors (Birks et al., 2017; Mammen et al., 2018; Minton et al., 2018). At a time of worldwide nurse shortages, this conflict and lack of support have a potentially negative impact on student attrition, with 
students at the very least choosing not to work in such environments or worse still, leaving the profession altogether (Fahie, \& McGillicuddy, 2018). The volume of responses suggests that student experience of WV remains at levels that are a cause for real concern. Whilst in the UK the profession has sought to introduce standards for learning in practice, unfortunately, this remains a 21 st-century problem. It is perhaps time for Higher Education Institutions to take joint action with healthcare providers to take a more serious look at the issue? The negative consequences of WV have been articulated and are reasons enough for a concerted effort toward this problem (Hills, 2018; Sun et al., 2018; Üzar-Özçetin et al., 2020). But there are also clear benefits for both placement providers and higher education institutions in proactively addressing the problem. If a placement provider develops a reputation for $\mathrm{WV}$, this will clearly deter people from wanting to work in that environment. Incidents of bullying and harassment toward staff could also be an indicator of a worrying culture and poor quality of care It is, of course, recognized that addressing cultural issues within a workplace can be a complex and time-consuming process but can have grave consequences if ignored (Morphet, Griffiths, Beattie, Reyes, \& Innes, 2018; Üzar-Özçetin et al., 2020; Yap, Kennerly, \& Flint, 2014).

A key finding from this study is the apparant normalization of poor behaviours within the workplaces. The prevalence of WV appears to have become routinely accepted and sustained as a 'normal part of the job'. Jiao et al. (2015) found that nurses expected violence as part of their job and consider being a target of violence to be 'normal', and with the resulting stigma and threat of further violence, preventing victims from reporting violent behaviour. More worryingly, the growing body of literature suggests WV is a widelyoccurring issue among nurses and nursing students around the World (Birks et al., 2017; Mammen et al., 2018; Minton et al., 2018; Üzar-Özçetin et al., 2020; Tee et al., 2016). So what can be done? 
Whilst placement providers and higher education institutions can initiate high level policies and procedures to help support students, this is unlikely to address the embedded cultural issues within clinical environments that students identify and feel powerless to change. This is where normalisation process theory (May \& Finch, 2009; Wood \& Cajkler, 2019) can help identify, think about, implement and evaluate new ways of thinking, working, or organising change in any work environment, including healthcare. The first stage is to acknowledge the issue to be addressed, in this case incidents of WV experienced by students, and the next is to encourage cognitive participation in the problem. This helps achieve ownership and engages the 'community' in seeking to solve the problem. This is followed by collective action and changes to working practices that are subsequently monitored and appraised. It may well be that placement providers identified as having a problem should be required to undertake these steps as part of an accreditation process for taking students within the clinical learning environment.

Perhaps, to further support and prepare students, undergraduate programs and continuing education for nurses should also include preparation and role-play simulations on how to handle WV, detailing the potential implications to improve the resilience of students (Martinez, 2017; Ming, Huang, Hung, Chang, Hsu, Tzeng, Huang, \& Hsu, 2019; SannerStiehr, 2017; Wu, Chen, Hsieh, Clinciu, \& Tung, 2019). A programme could consist of several stages. Firstly nurse educators should assist nursing students to develop an awareness of a violence culture and emphasise that WV is not 'normal' or 'part of the job'. Secondly, educators could highlight normalisation as the biggest risk that causes learned helplessness toward WV and help students to improve their coping skills through role play and use of reporting procedures. Lastly, educators (Adams, Knowles, Irons, Roddy, \& Ashworth, 2017; Caristo \& Clements, 2017) should give a clear message of zero-tolerance toward WV, no matter what the source, and remove offending clinical placements from training circuits until 
they have demonstrated that they have addressed the problem. By preparing both the students for the realities of practice and focusing on organisational cultures perhaps incidents of WV can be reduced and their impact on students learning be minimed so that over time WV towards healthcare students can be confined to history.

\section{Strengths and Limitations}

Typically research with students can present limitations when students feel inhibited to express their genuine thoughts or feelings because of an impact on lecturer-student relations. As our study was web-based, researchers had no direct contact with the students and so students were encouraged to express their experiences without feeling any pressure or anxiety which we believe improved the trustworthiness of our data. The use of SurveyMonkey helped prevent possible anxiety arising from unequal power dynamics. However, the response rate might be considered relatively small when compared with the total nursing students in the UK. The study is also limited by the sample size. To achieve saturation, the researchers analysed all data until all concepts were well-developed. Despite these potential limitations, the findings provide a rich picture of the perspectives of nursing students experiencing WV.

\section{CONCLUSION}

Many factors influence a nursing students' vision of the profession during their professional training and development and the majority finish with a positive view of the profession and high likelihood of employment. However, for a significant minority, the impact of WV can be extremely destructive for the individual, the profession and ultimately patient care with large numbers choosing to leave at a time when there is a national shortage of qualified staff. The undesirable truth is each year nearly more than half of the students face WV in environments where such behaviour becomes culturally embedded and passed between generations of nurses. This process of normalization requires intervention that will break this cycle to enable more appropriate workplace behaviours to flourish. 


\section{IMPLICATION FOR NURSING PRACTICE}

From the outset, it seems evident that an education provider who is not actively dealing with harassment and bullying might ultimately deter applicants and increase attrition. From an education provider perspective, there seems little point in trying to pretend such issues do not exist and so undergraduate curriculum and continuing education for nurses should include preparation and perhaps simulations with standardised patients on how to handle WV, detailing the potential implications to improve the resilience of students. It is only through such a concerted and transparent approach will we promote a more positive view of the profession. Universities, nurse managers and healthcare providers should work together to identify effective solutions that actively address the issues but that also build resilience so that students have the confidence to act against WV. We must collectively recognize that students are the future of nursing and have a vital role in developing and shaping the profession. If we want to eliminate $\mathrm{WV}$ we must model appropriate practice and communicate that such behaviours have no place in such a noble profession.

\section{Conflict of Interests}

No conflicts of interest.

\section{Funding}

None.

\section{REFERENCES}

Adams, J., Knowles, A., Irons, G., Roddy, A., \& Ashworth, J. (2017). Assessing the effectiveness of clinical education to reduce the frequency and recurrence of workplace violence. Australian Journal of Advanced Nursing, The, 34(3), 6.

Ahn, Y. H., \& Choi, J. (2019). Incivility experiences in clinical practicum education among nursing students. Nurse Education Today, 73, 48-53. 
Avander, K., Heikki, A., Bjerså, K., \& Engström, M. (2016). Trauma nurses' experience of workplace violence and threats: short-and long-term consequences in a Swedish setting. Journal of trauma nursing, 23(2), 51-57.

Becher, J. Visovsky C. (2012). Horizontal violence in nursing. MEDSURG Nurs, 21(4), 210 232.

Berry, P.A., Gillespie G.L., Gates, D., \& Schafer, J. (2012). Novice nurse productivity following workplace bullying. Journal of Nursing Scholarship, 44(1), 80-87.

Birks, M., Cant R., Budden, L., Russell-Westhead, M., Üzar Özçetin, Y.S. \& Tee, S. (2017). Uncovering degrees of workplace bullying: A comparison of baccalaureate nursing students' experiences during clinical placement in Australia and the UK. Nurse Education in Practice, 25, 14-21.

Caristo, J. M., \& Clements, P. T. (2019). Let's stop "eating our young": Zero-tolerance policies for bullying in nursing. Nursing2019 Critical Care, 14(4), 45-48.

Consolidated criteria for reporting qualitative research (COREQ): a 32-item checklist for interviews and focus groups. http://www.equatornetwork.org/reportingguidelines/coreq/. Accessed March 21, 2019.

Consolidated criteria for reporting qualitative research (COREQ): a 32-item checklist for interviews and focus groups. http://www.equator-network.org/reportingguidelines/coreq/. Accessed March 21, 2019.

Emanuel, V., \& Pryce-Miller, M. (2013). Creating supportive environments for students. Nursing Times; 37, 18-20.

Fahie, D., \& McGillicuddy, D. (2018). The (Un) Questionable Challenges of Sample Access, Recruitment and Retention in Contemporary Workplace Bullying Research. Concepts, Approaches and Methods, 1-30.

Flick, U. (2018). An introduction to qualitative research. UK: Sage Publications Limited. 
Fusch, P.I., \& Ness, L. R. (2015). Are we there yet? Data saturation in qualitative research. Qual Rep. 20(9), 1408-1416. $\quad$ Retrieved from https://nsuworks.nova.edu/tqr/vol20/iss9/3.

Gacki-Smith, J., Juarez, A.M., Boyett, L., Homeyer, C., Robinson, L., \& Maclean, S.L., (2009). Violence against nurses working in US emergency departments. Journal of Nursing Administration, 39, 340-349.

Gallacher, K., May, C. R., Montori, V. M., \& Mair, F. S. (2011). Understanding patients' experiences of treatment burden in chronic heart failure using normalization process theory. The Annals of Family Medicine, 9(3), 235-243.

Glomsås, H. S., Tranum, T. S., \& Johannessen, A. K. (2019). Piloting a practice model in a Norwegian nursing home-A student-managed ward: A way to empower students for the nursing role. Nurse Education in Practice, 34, 161-166.

Hartin, P., Birks, M., \& Lindsay, D. (2018). Bullying and the nursing profession in Australia: An integrative review of the literature. Collegian, 25(6), 613-619.

Heidegger, M. Being and time (trans. J, Stambaugh ). Albany, NY: SUNY Press, 2010.

Hills, D. J. (2018). Defining and classifying aggression and violence in health care work. Collegian, 25(6), 607-612.

Hills, D., Lam, L., \& Hills, S. (2018). Workplace aggression experiences and responses of Victorian nurses, midwives and care personnel. Collegian, 25(6), 575-582.

Hsieh, H.F., \& Shannon, S.E. (2005). Three approaches to qualitative content analysis. Qual. Health Res, 15(9), 1277-1288.

Islam, T., Ahmed, I., \& Ali, G. (2019). Effects of ethical leadership on bullying and voice behavior among nurses: mediating role of organizational identification, poor working condition and workload. Leadership in Health Services, 32(1), 2-17. 
Jenkins, C., \& Germaine, C. (2018). Solution-oriented learning to build resilience in mental health nursing students and recently qualified nurses. Mental Health Practice, 21(7).

Jiao, M., Ning, N., Li, Y., Gao, L., Cui, Y., Sun, H., Kang, Z., Liang, L., Wu, Q., \& Hao, Y. (2015). Workplace violence against nurses in Chinese hospitals: a cross-sectional survey. BMJ open, 5(3), e006719.

Laverty, S. M. (2003). Hermeneutic phenomenology and phenomenology: A comparison of historical and methodological considerations. International journal of qualitative methods, 2(3), 21-35.

Lopez, K. A., \& Willis, D. G. (2004). Descriptive versus interpretive phenomenology: their contributions to nursing knowledge. Qual Health Res, 14, 726-735.

Mackey, S. (2004). Phenomenological nursing research: methodological insights derived from Heidegger's interpretive phenomenology. Int J Nurs Stud, 42, 179-186.

Malterud, K., Siersma, V. D., Guassora, A. D. (2015). Sample size in qualitative interview studies guided by information power. Qual. Health Res. 26, 1753-1760.

Mammen, B., Hills, D. J., \& Lam, L. (2018). Newly qualified graduate nurses’ experiences of workplace incivility in Australian hospital settings. Collegian, 25(6), 591-599.

Manankil-Rankin, L., Lunyk Child, O., Chen, R., Martin, L., \& Bentley Poole, L. (2016). A Capstone Project: A way to Integrate Knowledge and Empower Students to Become Change Agents in the Practice Setting. Quality Advancement in Nursing EducationAvancées en Formation Infirmière, 2(1), 6.

Martinez, A. J. S. (2017). Implementing a workplace violence simulation for undergraduate nursing students: a pilot study. Journal of Psychosocial Nursing and Mental Health Services, 55(10), 39-44.

May, C., \& Finch, T., (2009). Implementing, embedding, and integrating practices: an outline of Normalization Process Theory. Sociology, 43(3), 535-554. 
Ming, J. L., Huang, H. M., Hung, S. P., Chang, C. I., Hsu, Y. S., Tzeng, Y. M., Huang, H. Y., \& Hsu, T. F. (2019). Using Simulation Training to Promote Nurses' Effective Handling of Workplace Violence: A Quasi-Experimental Study. International Journal of Environmental Research and Public Health, 16(19), 3648.

Minton, C., Birks, M., Cant, R., \& Budden, L. M. (2018). New Zealand nursing students' experience of bullying/harassment while on clinical placement: A cross-sectional survey. Collegian, 25(6), 583-589.

Morphet, J., Griffiths, D., Beattie, J., Reyes, D. V., \& Innes, K. (2018). Prevention and management of occupational violence and aggression in healthcare: A scoping review. Collegian, 25(6), 621-632.

Pryde, I. T. (2014). The dark side of nursing. Balboa Press, pp: 38-45, Bloomington.

Sanner-Stiehr, E. (2017). Using simulation to teach responses to lateral violence: guidelines for nurse educators. Nurse educator, 42(3), 133-137.

Sun, Y. Q., Ge, Y. X., Ke, Z. W., Li, Y. Y., Jin, Q. X., \& Lu, Y. F. (2018). Effect of workplace bullying on posttraumatic stress disorder in nursing staff. Zhonghua lao dong wei sheng zhi ye bing za zhi= Zhonghua laodong weisheng zhiyebing zazhi= Chinese journal of industrial hygiene and occupational diseases, 36(1), 22-25.

Tee, S., Üzar Özçetin, Y. S., \& Russell-Westhead, M. (2016). Workplace violence experienced by nursing students: A UK survey. Nurse Education Today, 41, 30-35.

Tee, S., Üzar Özçetin, Y. S., \& Russell-Westhead, M. (2017). Workplace violence in Nurse Education: An Issue of Workforce Retention - NET2017 Conference. https://www.advancehe.ac.uk/knowledge-hub/workplace-violence-nurse-educationissue-workforce-retention net2017-conference. Accessed September 21, 2019.

Üzar-Özçetin, Y. S., Tee, S., \& Erkan, M. (2020). A reputational risk for the profession: Workplace violence toward nursing students. Collegian. 
Wood, P., \& Cajkler, W. (2019). Normalisation Process Theory in ITE Lesson Study. In Lesson Study in Initial Teacher Education: Principles and Practices. Emerald Publishing Limited.

Wressell, J. A., Rasmussen, B., \& Driscoll, A. (2018). Exploring the workplace violence risk profile for remote area nurses and the impact of organisational culture and risk management strategy. Collegian, 25(6), 601-606.

Wu, J. C., Chen, H. Y., Hsieh, J. L., Clinciu, D. L., \& Tung, H. H. (2019). Enhancing health care personnel's response to ER violence using situational simulation. Clinical Simulation in Nursing, 28, 6-14.

Yap, T. L., Kennerly, S. M., \& Flint, E. P. (2014). Nursing culture assessment tool (NCAT): Empirical validation for use in long-term care. International Journal of Nursing Studies, 1, 241-249. 